\title{
Clonal Hematopoiesis and Acquired Thalassemia in Common Variable Immunodeficiency
}

\author{
Monika Belickova, ${ }^{* \dagger}$ Harry W. Schroeder, Jr., ${ }^{\dagger}$ Y. L. Guan, ${ }^{\dagger}$ \\ Joseph Brierre, ${ }^{+}$Seth Berney, ${ }^{\dagger}$ Max D. Cooper, ${ }^{\neq+}$ \\ and Josef T. Prchal ${ }^{\dagger}$ \\ *Prague Institute of Hematology, Czech Republic. \\ tThe Divisions of Hematology/Oncology and Developmental and \\ Clinical Immunology and ₹The Howard Hughes Medical Institute \\ Department of Medicine, University of Alabama at Birmingham, \\ Birmingham, Alabama, U.S.A.
}

\begin{abstract}
Background: Common variable immunodeficiency (CVID) is defined by hypogammaglobulinemia and increased susceptibility to infections. The gene defect responsible for CVID remains unknown.

Methods: During the course of their CVID disease, a female and three male patients developed microcytic anemia. The investigation of this anemia forms the basis for this report.

Results: Reticulocyte globin chain synthesis studies revealed the abnormal $\alpha / \beta$ ratios that are pathognomonic of thalassemia. Through transcriptional analysis
\end{abstract}

of the glucose-6-phosphate-dehydrogenase (G6PD) locus of the active $\mathrm{X}$-chromosome in blood cells, we determined that the female patient has clonal reticulocytes, platelets, granulocytes, and B and T lymphocytes.

Conclusions: The simultaneous presence of globin synthesis abnormalities and panhypogammaglobulinemia suggests that a common insult at the stem cell level could contribute to the development of CVID and acquired thalassemia.

\section{INTRODUCTION}

CVID, defined by panhypogammaglobulinemia and increased susceptibility to infections, has long been considered a category encompassing a heterogeneous group of primary immune system disorders. Most CVID patients exhibit a distinctive phenotype characterized by normal numbers of immunoglobulin-bearing $B$ cell precursors but a deficiency of all immunoglobulin isotypes due to an arrest in plasma cell differentiation (reviewed in (1)). Among a large group of CVID- and IgA-deficient patients, we observed that approximately two-thirds were associated with two ancestral extended MHC haplotypes, termed Haplotype I (HLA-DQB $1 * 0201$, HLA-DR

Address correspondence and reprint requests to: Josef $\mathrm{T}$. Prchal, Division of Hematology/Oncology, 513 Tinsley Harrison Towers, UAB Station, University of Alabama at Birmingham, Birmingham, AL 35294, U.S.A.
3, C4B-Sf, C4A-O, G11-15, Bf-0.4, C2-a, HSP7.5, TNF $\alpha-5$, HLA-B8, HLA-Al) and Haplotype II (HLA-DQB 1*0201， HLA-DR7，C4B-S，C4A-L, G1 1-4.5, Bf-0.6, C2-b, HSP-9, TNF $\alpha-9$, HLAB44) (1). The phenotypic similarities between selective IgA deficiency (IgAD) and CVID, the association of the same MHC haplotypes with both disorders (2), and the intrafamilial occurrence of $\operatorname{IgAD}$ and CVID support the idea that IgAD and CVID may represent polar ends of a clinical spectrum of immunodeficiency that reflects a shared genetic susceptibility (2).

$\mathrm{Hb} \mathrm{A}$ is a tetrameric molecule formed by $\alpha$ and $\beta$-globin chains. Under normal circumstances, reticulocyte production of $\alpha$ - and $\beta$-globin is perfectly balanced (3). Imbalanced synthesis in thalassemias leads to free globin chains that are toxic to erythroblasts. This results in the production of smaller than normal red cells and decreased mean corpuscular volume (MCV). The 
vast majority of thalassemic patients are of Mediterranean, African, and South Asian extraction who inherit the trait in a Mendelian dominant form. The globin gene defects responsible for thalassemias include intron splicing mutations, stop codon mutations, gene deletions, and mutations that interfere with globin transcription (3). In addition to the classic forms of thalassemia, there are scattered reports of individuals with an acquired form of the disease. Although the etiology of these acquired thalassemias is unknown, these patients typically suffer from leukemia, aplastic anemia, myelodysplastic syndromes, or other myeloproliferative disorders (4-6). All of these conditions are characterized by clonally restricted hematopoiesis, suggesting that acquired thalassemia may result from somatic mutations in a progenitor stem cell.

Hematological abnormalities are frequently observed in our patients with CVID. Among six CVID patients evaluated for anemia, we identified four individuals with an acquired imbalance of globin chain synthesis. Clonality studies of hematopoiesis in one of the female patients with acquired thalassemia revealed restricted clonality of her reticulocytes, platelets, granulocytes, and $\mathrm{B}$ and $\mathrm{T}$ lymphocytes, thus suggesting a possible link between these acquired lymphocyte and erythrocyte disorders.

\section{MATERIALS AND METHODS}

\section{Subjects}

Three males and three females with CVID were evaluated with informed consent according to a research protocol approved by the University of Alabama at Birmingham Human Use Institutional Review Board.

\section{Hemoglobinopathy Evaluation}

Hemoglobinopathy evaluation, including globin chain synthesis, was performed using routine clinical laboratory procedures previously described (7).

\section{Cell Preparations and DNA Studies}

For clonality studies, skin fibroblasts and myeloid cells were obtained and separated as previously described (8). Lymphocyte subpopulations were isolated from peripheral blood by flow cytometric sorting of the nonadherent mononuclear cells for CD19 (B lymphocytes) and CD3 (T lymphocytes) (8). Hair was plucked from the scalp, and the roots were placed in sterile microtubes. The buccal mucosa was scrubbed with a sterile tongue depressor to obtain epithelial cells (8). Ligase detection reaction (LDR) analysis of the common nucleotide \#1311 (C/T) exonic polymorphism of the X-linked gene G6PD was performed using $10 \mathrm{ng}$ of genomic DNA or cDNA template prepared from total cellular RNA (8). Thermostable ligase (a kind gift of Dr. Francis Barany (9)) was diluted in $10 \mathrm{mM}$ Tris $\mathrm{HCl}, \mathrm{pH}$ 8.0, in $50 \%$ glycerol containing $0.5 \mathrm{mM}$ EDTA, 1 $\mathrm{mM}$ dithiothreitol, $100 \mu \mathrm{g}$ of bovine serum albumin (Fraction V), $0.1 \%$ Triton X-100, and 1.0 $\mathrm{mM} \mathrm{NAD}{ }^{+}$. After 30 cycles of $1 \mathrm{~min}$ at $94^{\circ} \mathrm{C}$ and $4 \mathrm{~min}$ at $65^{\circ} \mathrm{C}(10)$, the reaction products were separated on $10 \%$ polyacrylamide gels containing $7 \mathrm{M}$ urea and detected by autoradiography.

\section{RESULTS}

Patient 1 (identification \#S23.7), a male of Anglo-Saxon and American Indian extraction, had an MCV of 80 with a hemoglobin of $14.0 \mathrm{gm} / \mathrm{dl}$ when seen for headache at age 18. Recurrent sinopulmonary infections began at age 25 , and panhypogammaglobinemia was discovered at age 27 when immunoglobulin replacement therapy was instituted. The patient developed a severe microcytic anemia at age 30 (see Table 1) and at age 32 acquired progressive lymphadenopathy, which was due to a large cell follicular lymphoma of B-lineage cells. Hemoglobin electrophoresis was normal (see Table 1), and iron deficiency and sideroblastic anemias were excluded by biochemical studies and bone marrow morphology. Analysis of reticulocyte globin synthesis revealed an $\alpha / \beta$ globin synthetic ratio of 1.4 (this ratio remained unchanged when repeated 3 weeks later at 1.42). This was diagnosed as an acquired $\beta$ thalassemia, since both of his parents had normal $\beta$-globin synthetic ratios and no evidence of red cell microcytosis or anemia (see Table 1). The proband and his mother have the HLA DR7-B44 extended haplotype (Haplotype II) previously found in one-third of our CVID patients (2). The mother and father had normal serum immunoglobulin levels, and the father's paternity was confirmed by HLA studies.

Patient 2 (S25.59), a 26-year-old Caucasian female, began to experience recurrent sinopulmonary infections in her late teens, and panhypogammaglobinemia was discovered at age 23 . 


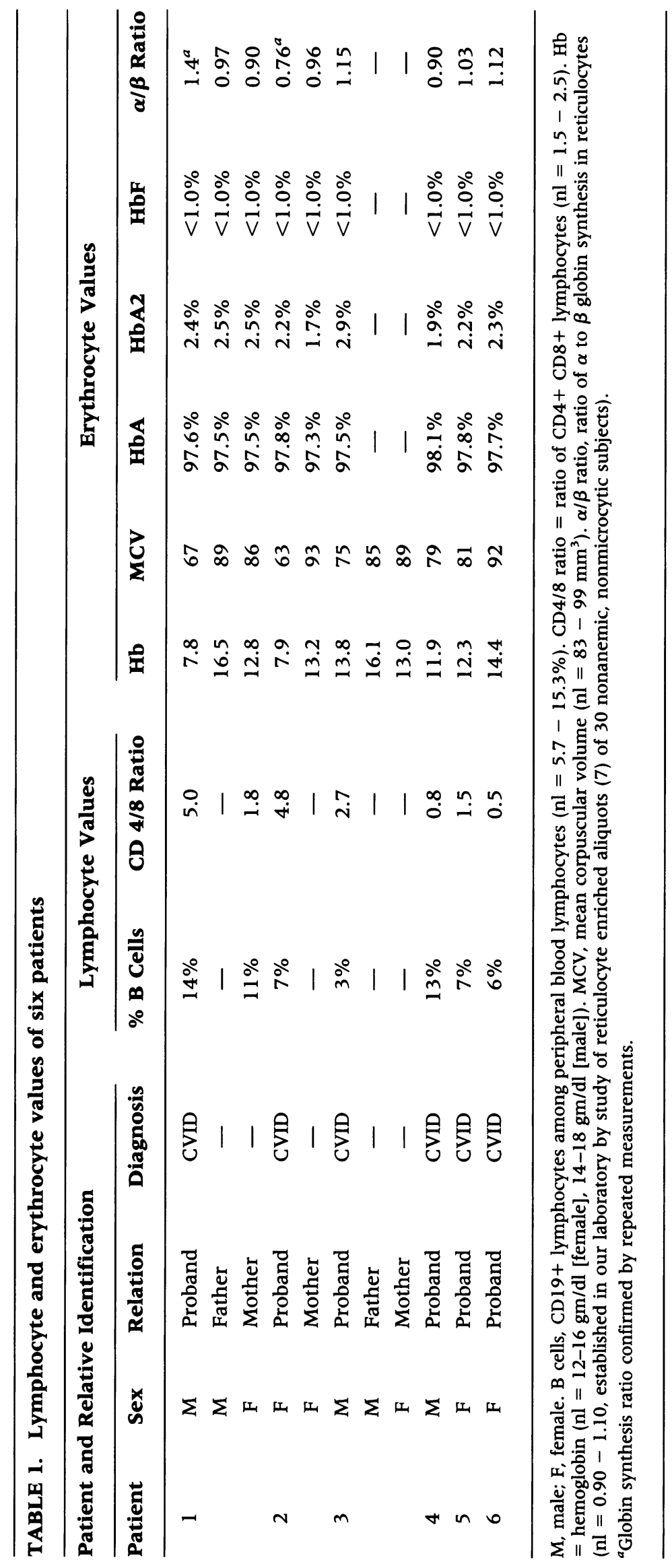


She has inherited neither of the two MHC haplotypes associated with CVID in our patient population (2). At the time of diagnosis of CVID she was anemic and microcytic (see Table 1), but 12 years prior to diagnosis she was not anemic and 5 years prior to diagnosis she had normocytic erythrocyte indices. Iron deficiency and sideroblastic anemias were excluded by serum iron and ferritin studies and bone marrow evaluation. The diagnosis of $\alpha$ thalassemia was established by an $\alpha / \beta$ globin synthetic ratio of 0.76 (this ratio remained unchanged when repeated 8 weeks later at 0.77$)$. Her mother has normal red cell values (Table 1). Her father is deceased.

Two of 31 additional patients with CVID in our study population (2) had microcytic erythrocytes $\left(\mathrm{MCV}<80 \mu \mathrm{m}^{3}\right.$ ). Patient 3 (S26.12), a 22-year-old Caucasian male has had microcytic anemia since age 12, when he was discovered to have CVID (Table 1). Patient 4 (S4.1), a 68-yearold Caucasian male, was only mildly anemic at age 63 with a hemoglobin of $13 \mathrm{~g} / \mathrm{dl}$ and an MCV of 81 and now has moderate microcytic anemia (MCV of 79 and a hemoglobin of $11.9 \mathrm{~g} / \mathrm{dl}$ ). Neither Patient 3 nor Patient 4 has the MHC Háplotypes I or II. Hemoglobinopathy evaluation, including bone marrow analysis, revealed evidence of $\beta$ thalassemia in Patient 3 (Table 1) and a borderline globin synthetic value in Patient 4. Like Patients 1 and 2, Patients 3 and 4 have a normal proportion of the minor hemoglobins. The parents of Patient 3 exhibited normal hemoglobin concentrations and red cell indices, but both parents of Patient 4 are deceased (Table 1).

Two additional female CVID patients with $\mathrm{MCV}<80$ were selected at random for globin chain synthesis evaluation. Patient 5 (S27.31) has Haplotype II, whereas Patient 6 (S9.4) has neither common CVID associated haplotype. In Patient 5, the globin synthetic studies were normal while Patient 6 (S9.4) had borderline $\beta$ thalassemic globin synthetic values. Unfortunately, neither female was informative for the G6PD polymorphism (see below).

In order to search for a common element in the pathogenesis of the acquired erythrocyte and lymphocyte defects in these patients, we performed clonality studies of mature hematopoietic progenitors in both thalassemic females. By examination of genomic DNA, only Patient 2 was found to be heterozygous for the X-chromosome G6PD \#1311 C/T exonic polymorphism (7). As shown in Fig. 1A, analysis of reverse transcribed mRNA from isolated myeloid cells (reticulocytes
$R$, granulocytes $G$, and platelets $P$ ) and the circulating peripheral blood mononuclear cells $(\mathrm{M})$ revealed only a single $C 44$ bp allelic transcript. In contrast, the nonhematopoietic cells (hair root $\mathrm{H}$, buccal epithelial mucosal cells $\mathrm{Me}$, and skin fibroblasts S) all showed the allelic $46 \mathrm{bp} \mathrm{T}$ and $44 \mathrm{bp} \mathrm{C}$ transcripts from both $\mathrm{X}$ chromosomes (Fig. 1A (10)). Analysis of T lymphocytes that had been isolated 1 year prior to the current study revealed that a small proportion of Patient 2 's circulating $\mathrm{T}$ lymphocytes expressed the allelic $46 \mathrm{bp} T$ transcript (Fig. 1A). When B and T cell populations were isolated from the current mononuclear cell fraction, rtPCR LDR analyses (Fig. 1B) revealed the presence of only a single allele, suggesting that the proportion of the nonclonal T-cell population expressing the $46 \mathrm{bp} \mathrm{T}$ transcripts has disappeared over time.

\section{DISCUSSION}

This study establishes the presence of thalassemia in a significant proportion of patients with CVID. A review of previous red cell studies and an analysis of family members documented the acquired nature of this red cell disorder. The signs and symptoms of clonal disorders of hematopoiesis, such as myeloproliferative disorders, leukemias, and aplastic anemia, are due to defects in cells at the terminal stages of differentiation, but owe their genesis to mutation(s) at a progenitor cell level.

In females, one of the two $\mathrm{X}$ chromosomes is randomly inactivated early in ontogeny. The $X$ chromosome inactivation phenomenon allows the detection of clonal hematopoiesis, since the active $\mathrm{X}$ chromosome will be the same in all the offspring of an altered progenitor cell. We have recently described an assay for the transcriptional polymorphism of $\mathrm{X}$ chromosome transcripts derived from the G6PD gene (8), in which an exonic polymorphism $(\mathrm{C} / \mathrm{T})$ exists at position 1311 in all ethnic and racial groups. While the ratio of these transcripts is variable because of the random nature of $X$ chromosome inactivation, we have not encountered a single allelic transcript in any normal tissue in contrast to the presence of a single transcript present in clonally derived tissues in the heterozygous women whom we have studied to date.

Using the ligase detection reaction, we were able to demonstrate clonal hematopoietic progeny of both myeloid and lymphoid cells in a patient with CVID and acquired thalassemia. 

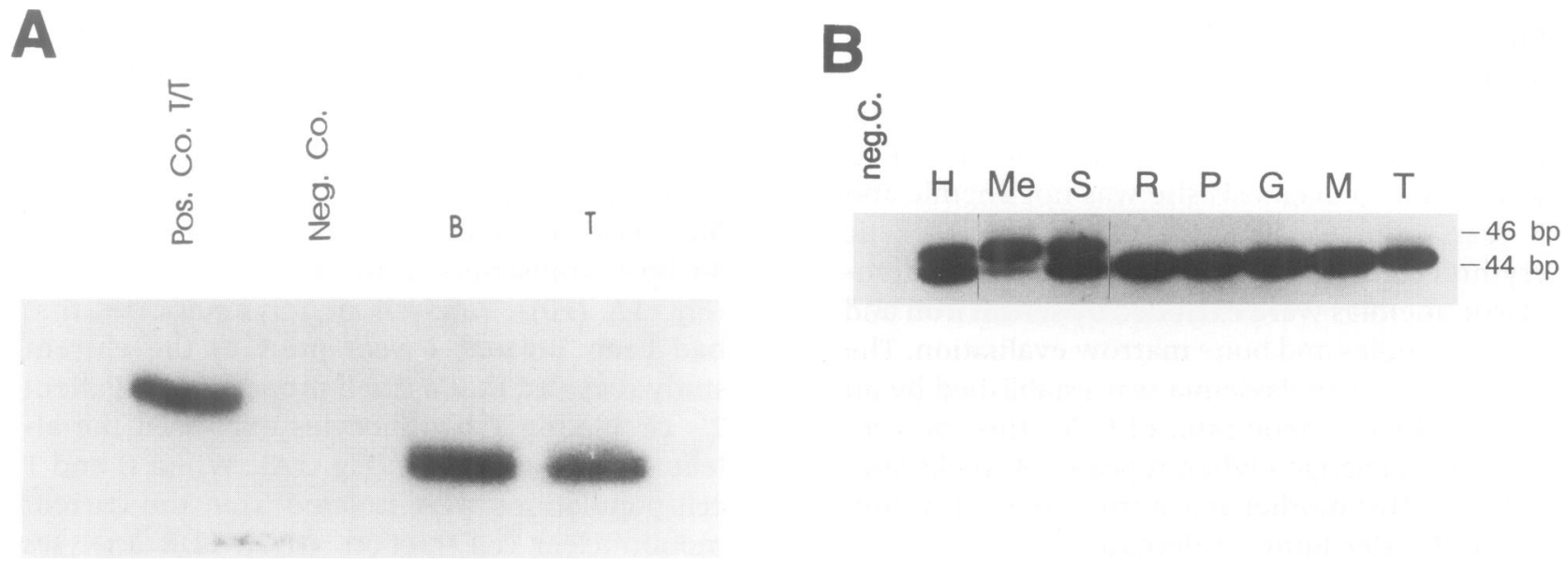

FIG. 1. X chromosome usage by hemopoietic and nonhemopoietic cells in a female with CVID and acquired thalassemia analyzed by detection of glucose-6-phosphate dehydration (G6PD) gene polymorphism

(A) The ligase detection analysis (LDR) of a G6PD polymorphism (C/T exonic nucleotide \#1311) (8) employed a cDNA prepared by reverse PCR reaction from total cellular RNA (10). The negative control (Neg. Co.) represents a sham experiment without added DNA template. Hair root follicles (H), mouth epithelial cells (Me), skin cultured fibroblasts $(S)$, reticulocytes $(R)$, platelets $(P)$, granulocytes $(G)$, mononuclear cells $(M)$, and T-lymphocytes (T), of a female with CVID (Patient 1) were examined using a cDNA template prepared reaction from total cellular RNA. (B) The LDR analysis employed a positive T/T control (Pos. Co.) reaction with added DNA template from an individual homozygous for the T polymorphism), and as a negative control (Neg. Co.) a sham experiment without added DNA template. B and T lymphocytes were obtained by immunofluorescence sorting of peripheral blood mononuclear cells as described in Methods. Detection of an allelic transcript of an active X chromosome was done as described $(8,10)$.

Thus we propose that the acquired abnormality in globin synthesis and lymphocyte differentiation seen in these patients may reflect a clonal hemopoietic disorder. In Wiskott Aldrich Syndrome (11), X-linked agammaglobulinemia (12), and some other X-linked disorders (13), carrier females demonstrate clonality in the affected cell lineages, presumably due to selection against the progeny of cells that express a deleterious gene. The possibility of this type of "cell selection" in this subset of CVID patients cannot be ruled out at present. However, the development of a B cell lymphoma in our index Patient 1 would be in keeping with the malignant transformation frequently seen in premalignant clonal stem cell disorders, such as the blastic transformation of chronic myelogeneous leukemia.

The molecular mechanisms leading to the acquired differentiation defect of B lymphocytes and the acquired abnormality of globin synthesis in these thalassemic CVID patients remain unknown. The regulation of expression and transcription of the globin genes is better understood at the molecular level than that of most other human genes (14). Interestingly, regulation of globin transcription is mediated by factors that are not always erythroid specific but also operate on nonglobin genes in B cells and other tissues (15). It could be hypothesized that a gene mutation in the hematopoietic pluripotent stem cell, the precursor of both lymphocytes and erythrocytes, could be associated with an abnormality of a transcription or regulatory factor that plays a major role in both globin gene expression and in B lymphocyte differentiation. Identification of this putative factor responsible for the acquired thalassemia may result not only in the understanding of one potential etiology for CVID but also in a better understanding of normal erythrocyte and lymphocyte differentiation.

\section{ACKNOWLEDGMENTS}

This work was supported, in part, by a VAH Merit grant (J.T.P.) and United States Public Health Service Grants IROI HL51650 and HL50077 (J.T.P.), AR03555 (H.W.S.), AI30879, and AI34568, AR20614 (H.W.S.), AI07051 (S.B.), and AI33621 (H.W.S.). The work was 
conducted through the General Clinical Research Center of the University of Alabama at Birmingham, supported by USPHS grant MOI RR00032.

\section{REFERENCES}

1. (1992) Primary immunodeficiency diseases: Report of a WHO scientific group. Immunodef. Rev. 3: 195-236.

2. Volanakis JE, Zhu ZB, Schaffer FM, et al. (1992) Major histocompatibility complex class III genes and susceptibility to immunoglobulin A deficiency and common variable immunodeficiency. J. Clin. Invest. 89: 19141922.

3. Bunn HF, Forget BG. (1986) Hemoglobin: Molecular, Genetic and Clinical Aspects. WB Saunders, Philadelphia.

4. Anagnou NP, Ley TJ, Chesbro B, et al. (1983) Acquired $\alpha$-thalassemia in preleukemia is due to decreased expression of all four $\alpha$-globin genes. Proc. Natl. Acad. Sci. U.S.A. 80: 6051-6055.

5. Higgs DR, Wood WG, Barton C, Weatherall DJ. (1983) Clinical features and molecular analysis of acquired hemoglobin $\mathrm{H}$ disease. Am. J. Med. 69: 181-184.

6. Hoyle C, Kaeda J, Leslie J, Luzzatto L. (1991) Acquired $\beta$ thalassemia trait in myelodysplastic syndrome. Brit. J. Med. 79: 116-131.

7. Prchal JT, Scott CW, Rahbar S, et al. (1989) Mild sickle cell anemia associated with $\alpha$ globin mutant $\alpha$-Montgomery. Am. J. Med. 75: 232-236.
8. Prchal JT, Prchal JF, Barany F, Guan YL. (1993) Transcriptional analysis of the active $\mathrm{X}$-chromosome in normal and clonal hematopoiesis. Blood 81: 269-272.

9. Barany F, Gelfand E. (1991) Cloning overexpression and nucleotide sequence of a thermostable DNA ligase-encoding gene. Gene 109: 1-11.

10. Prchal JT, Guan YL. (1993) A novel clonality assay based on transcriptional analysis of the active X chromosome. Stem Cells 11: 62-65.

11. Conley ME, Brown P, Pickard RA. (1986) Expression of the gene defect in X-linked agammaglobulinemia. N. Engl. J. Med. 315: 564-568.

12. Prchal JT, Carroll AJ, Prchal JF, et al. (1980) Wiscott-Aldrich Syndrome: Cellular impairments and their implication for carrier detection. Blood 56: 1048-1054.

13. Nyhan WL, Bakay B, Connor JD, Marks JF, Keele DK. (1970) Hemizygous expression of glucose-6-phosphatedehydrogenaseinerythrocytes of a heterozygotes for the Lesch-Nyhan syndrome. Proc. Natl. Acad. Sci. USA 65: 214-219.

14. Felsenfeld G. (1992) Chromatin structure and globin transcription. Nature 355: 219224.

15. Peters LL, Andrews NC, Eicher EM, et al. (1993) Mouse microcytic anemia caused by a defect in the gene encoding the globin enhancer-binding protein NF-E2. Nature 1362: $768-770$. 